Supplement of The Cryosphere, 13, 1-20, 2019 https://doi.org/10.5194/tc-13-1-2019-supplement (C) Author(s) 2019. This work is distributed under the Creative Commons Attribution 4.0 License.

(c) (1)

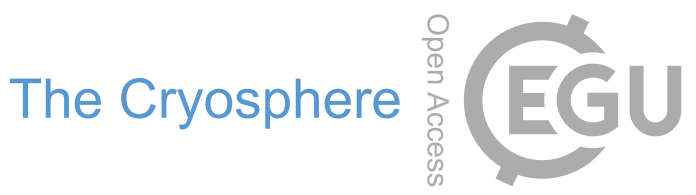

Supplement of

\title{
Definition differences and internal variability affect the simulated Arctic sea ice melt season
}

\author{
Abigail Smith and Alexandra Jahn \\ Correspondence to: Abigail Smith (abigail.1.smith@ colorado.edu)
}

The copyright of individual parts of the supplement might differ from the CC BY 4.0 License. 


\section{Supplementary Material}

\section{Melt and freeze onset definition development}

The diagnosed melt and freeze onset were found to be impacted by three main decisions in the definition development, which are detailed below.

The first decision was which days of the year to check for melt and freeze onset. For each model year and definition, we begin checking for melt onset on January 1 and stop checking on December 31st. However, capturing the timing of freeze onset is less straightforward. If we were to begin checking for freeze onset on January 1st, our definition criteria would be met during the late freeze period of January-April rather than the fall freeze-up period. One solution would be to begin checking for freeze onset starting on the day of melt onset. However, because we want to assess the impact of definition choices on melt and freeze onset separately, and because the best definition to use is application-dependent, we keep the definitions of melt and freeze onset independent of each other. By beginning the check for freeze onset on a common date for each definition, we are able to create melt season length definitions using different combinations of melt and freeze onset definitions, as described in 2.3. Furthermore, we need to account for the fact that by the middle of the 21 st century, projections of freeze onset indicate that large portions of the Arctic may not freeze until the the following year. For these reasons, we begin checking for freeze onset on June 29th (day 180) each year and stop checking on May 15th of the next year (day 500). These dates are based on our analysis of changes in the percent area where melt and freeze onset conditions are met due to start and end dates. For example, by beginning the check for freeze onset two weeks later using the surface temperature definition, the area of missing values (where freeze onset occurred before the start date) increases by as much $23 \%$ in some years during the satellite era.

The second decision was the threshold each variable must pass in order to define the start of sea ice melting or freezing. Here, we compared what makes sense physically (i.e., ice growth becoming nonzero) and whether these thresholds provide sensible continuous melt and freeze onset dates, based on the annual cycle of the variables at selcted points in the Arctic. For definitions using thermodynamic variables, the zero-line makes the most physical sense and provides reasonable melt and freeze onset dates, albeit earlier/later than surface-based definitions of melt/freeze onset. In the frazil freeze onset definition, 0 $\mathrm{cm} /$ day is also found to be both physically sensible and provide appropriate freeze onset dates. However, in the snowmelt melt onset definition and the congelation freeze onset definition, $0.01 \mathrm{~cm} /$ day is a more appropriate threshold to capture continuous melt and freeze onset than $0 \mathrm{~cm} / \mathrm{day}$, as each of these variables show very small $\left(10^{-7} \mathrm{~cm} /\right.$ day $)$, nonzero values throughout many times of the year.

The seasonal cycle of surface temperature in the CESM LE is more variable than the sea ice variables. It is also less directly related to sea ice growth and loss, so choosing a threshold that is representative of continuous melt and freeze onset is more complex. For melt onset, $-1{ }^{\circ} \mathrm{C}$ has been previously used as a threshold (Jahn et al., 2012; Mortin and Graversen, 2014). Other options include the melting point of ice $\left(0^{\circ} \mathrm{C}\right)$ and the observed surface air temperature at which sea ice melts in the Arctic (around $-1.9^{\circ} \mathrm{C}$ ) (Mortin and Graversen, 2014; Andreas et al., 1982). Our sensitivity tests show that out of the threshold choices of $0{ }^{\circ} \mathrm{C},-1{ }^{\circ} \mathrm{C}$, and $-1.9{ }^{\circ} \mathrm{C}$, pan-Arctic averages of the $-1{ }^{\circ} \mathrm{C}$ surface temperature definition had the least daily variability, allowing us to use a shorter timeframe for this definition (see next paragraph). Thus we adopt a threshold of -1 
${ }^{\circ} \mathrm{C}$ in our surface temperature melt onset definition. When defining freeze onset, we are primarily concerned with the freezing point of salt water. We therefore use the threshold $-1.8^{\circ} \mathrm{C}$ for the surface temperature definition of freeze onset. This threshold is equal to the constant freezing point of seawater used in the CESM LE, which means that definitions developed in models with salinity-dependent freezing points (for example the new CESM2) may find other thresholds more appropriate.

The third key decision for the melt and freeze onset definitions was how many consecutive days each variable must be above or below the threshold in order to constitute continuous melt or freeze (referred to here as the "timeframe"). We analyzed the seasonal cycle of each variable at multiple grid cells throughout the Arctic to determine each variable's behavior during seasonal transitions. Because the annual cycle of each variable is not the same at every grid cell every year, the choice of timeframe must accurately capture continuous melt and freeze while still taking into account that synoptic meteorological events can push variables above/below the chosen threshold defined for melt and freeze for short periods of time (Persson, 2012). For each definition, the first day of the timeframe is chosen as the date of melt or freeze onset.

We find that for a majority of the definitions, three days is a sufficient middle ground that produces melt and freeze onset for at least $90 \%$ of the Arctic in most years. However, melt and freeze onset sensitivity to the timeframe depends on the variable used in the definition. Surface temperature and snowmelt are very sensitive to the timeframe, frazil ice growth is somewhat sensitive to the timeframe and congelation ice growth and thermodynamic ice volume tendency are not sensitive to the timeframe at all. Due to large temperature variability during the fall in the Arctic, continuous freeze onset based on surface temperature is most accurately captured through a timescale of 21 days. A timeframe of three days has previously been used to determine continuous snowmelt using snowmelt runoff in the Canadian Coupled Global Climate Model (CGCM3) (Wang et al., 2011). We found 5 days to be an appropriate timeframe for continuous melt onset in the CESM LE using snowmelt, 20 as the average pan-Arctic melt onset is roughly 20 days earlier when using 3 days, possibly capturing early melt events. The snowmelt melt onset and surface temperature freeze onset definitions are therefore exceptions to the 3-day timeframe.

Another approach to capturing continuous melt and freeze onset is to use smoothing techniques such as running means or medians. Mortin and Graversen (2014) use a 14-day running median of their chosen model variable, surface air temperature, to exclude early or transitory melt and freeze events from their analysis. Holland and Landrum (2015) use a 15-day running mean of snow thickness to define melt onset in the CESM LE and CMIP5 models. Smoothing techniques are not employed in our study, as running medians and means were found to excessively reduce the number of times that the melt and freeze onset criteria were met for some variables. For example, 14-day running medians and means were found to reduce the number of times that the melt onset criteria was met using the CESM LE surface temperature definition over the satellite era by $8 \%$ and $18 \%$ of the area in the Arctic, respectively. Sensitivity of the melt and freeze onset definitions to running medians/means depends on the variable, and it has a smaller impact on the snowmelt definition $(\sim 5 \%)$ and almost no impact on the thermodynamic ice volume tendency definition. 


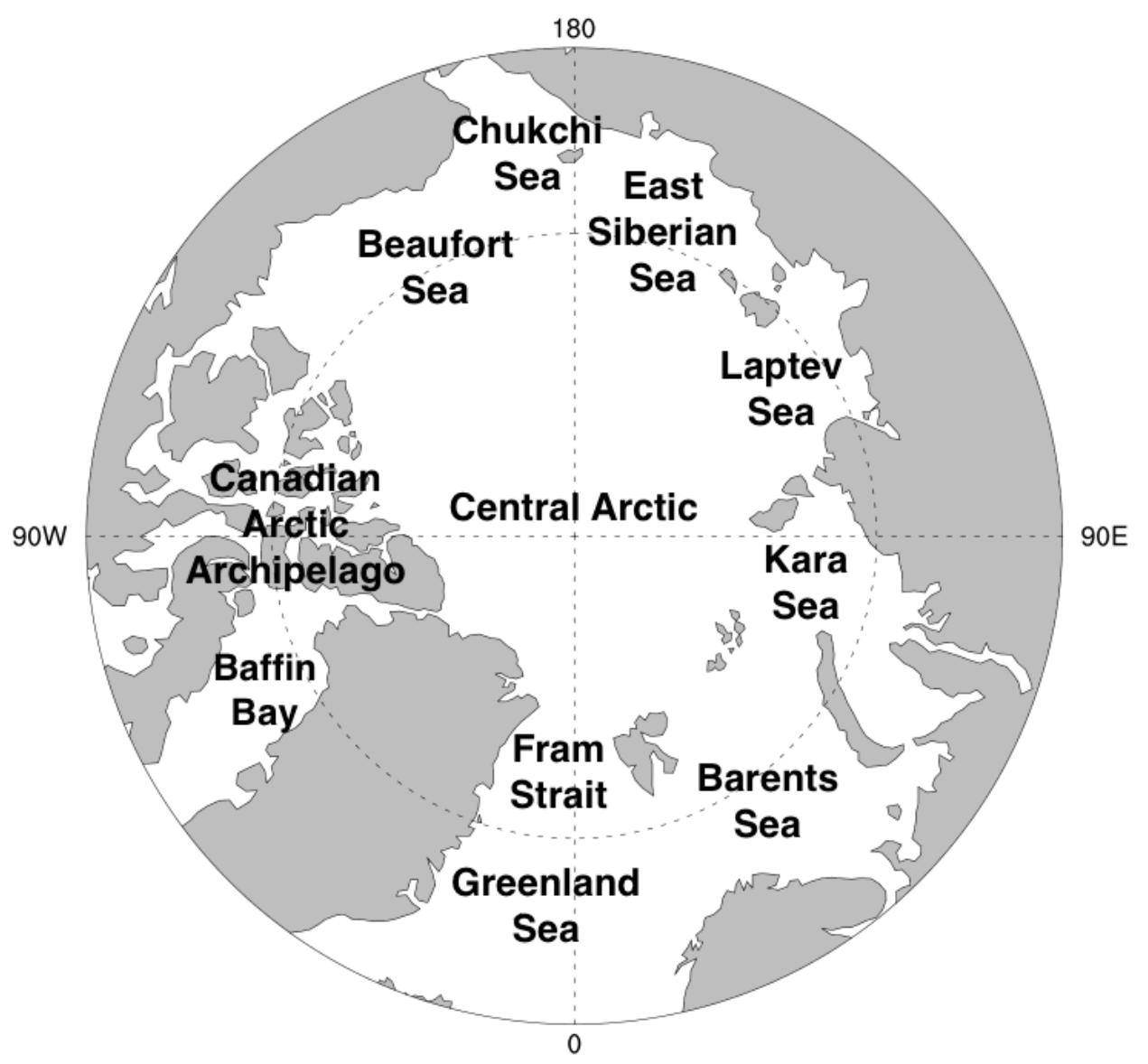

Figure S.1. Map of most major Arctic seas and features north of $66^{\circ} \mathrm{N}$. 

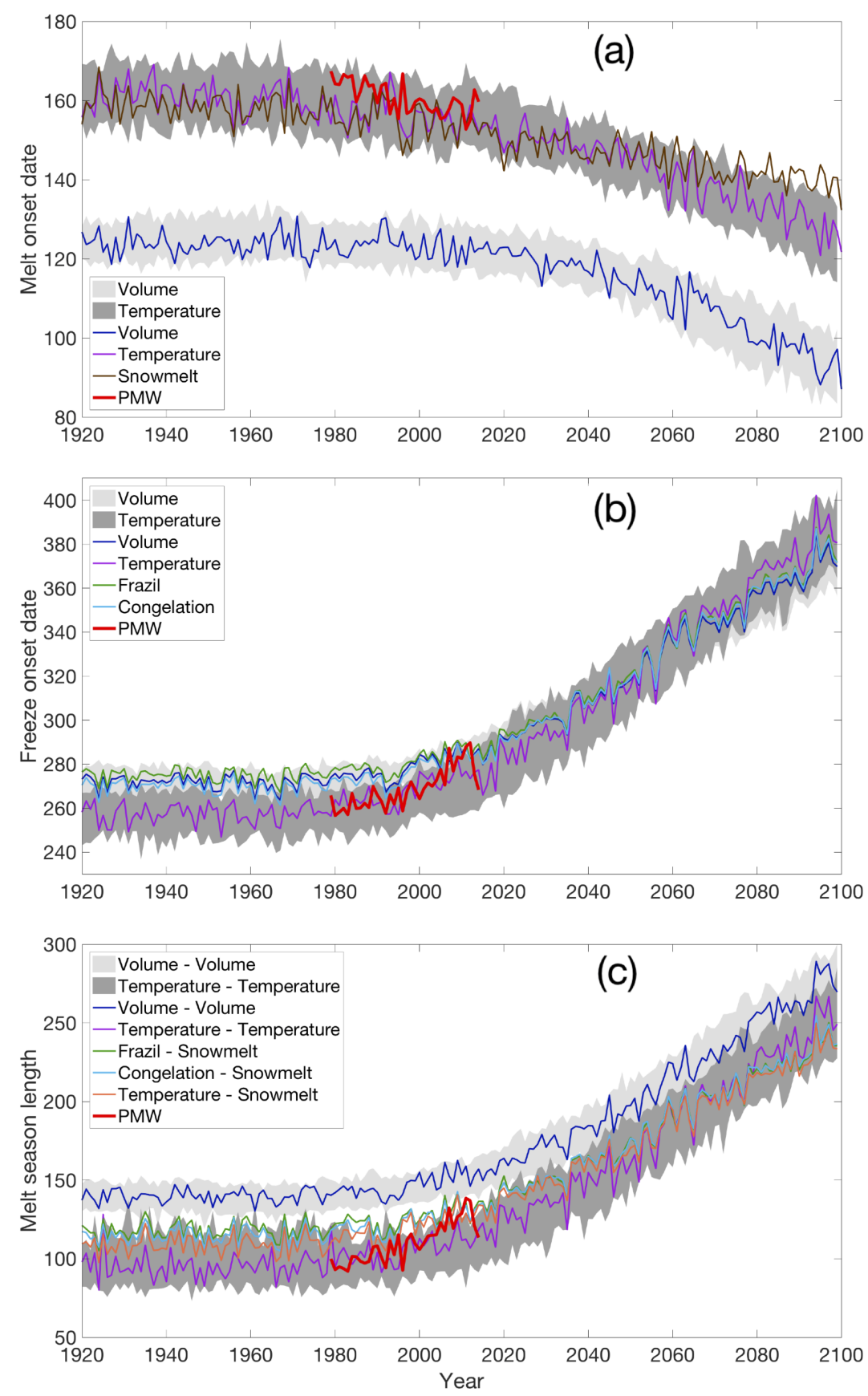

Figure S.2. Same as Fig. 1, but for ensemble member 34. Melt season characteristics averaged over $66^{\circ} \mathrm{N}$ to $84.5^{\circ} \mathrm{N}$ for PMW satellite observations and each CESM LE definition for (a) melt onset (b) freeze onset and (c) melt season length. PMW satellite observations are shown in red. Other colored lines represent ensemble member 34 and the gray shading represents the ensemble spread, for the two definitions (surface temperature and thermodynamic ice volume tendency) that have 40 ensemble members. 

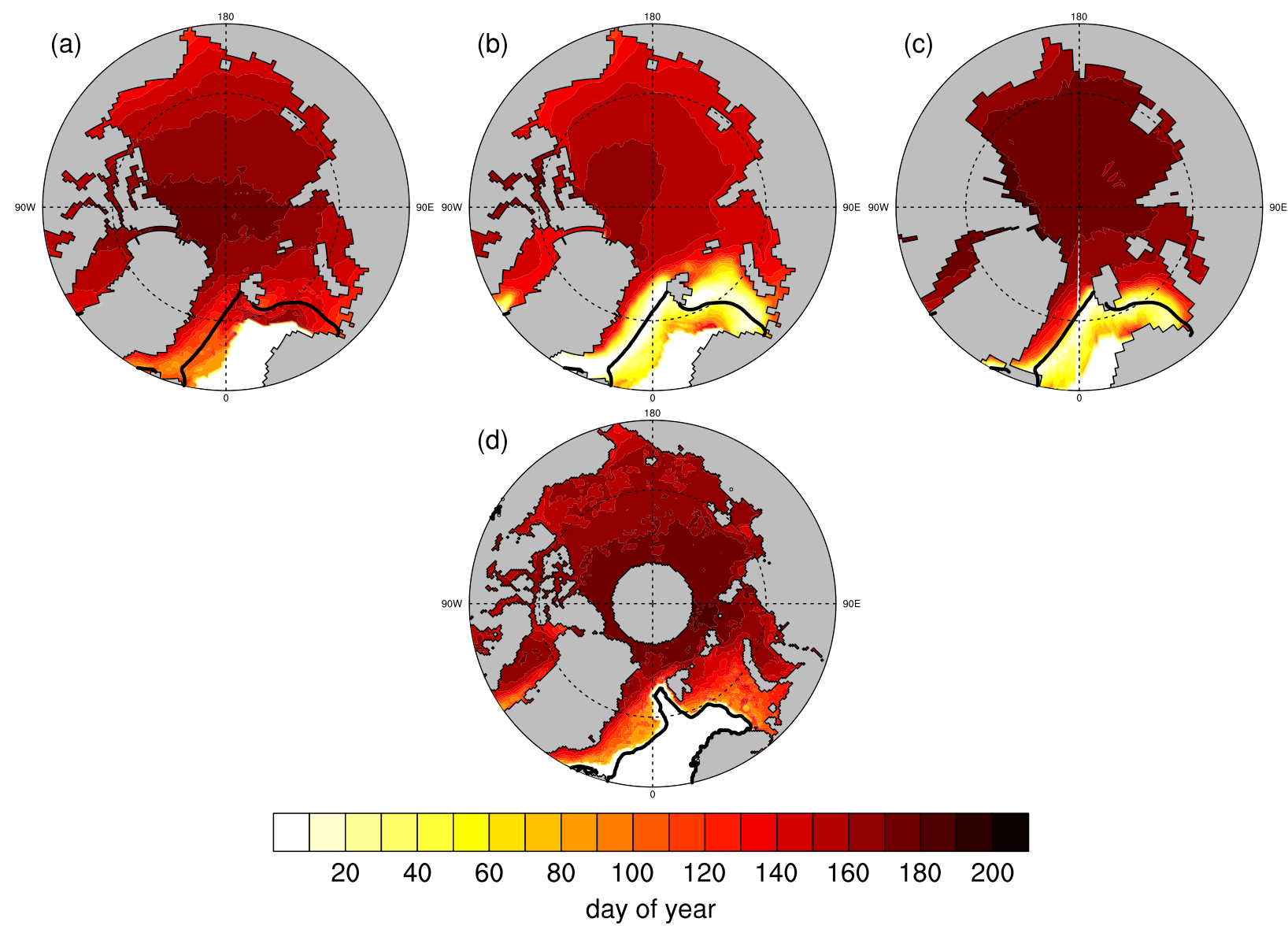

Figure S.3. Same as Fig. 3, but for ensemble member 34. Average melt onset dates over 1979-2014 for each CESM LE definition using ensemble member 34: (a) snowmelt definition (b) thermodynamic ice volume tendency definition (c) surface temperature definition and (d) PMW satellite observations. The black line denotes the mean March ice edge (15\% ice concentration) from 1979-2014 using the CESM LE (a-c) and NSIDC Bootstrap (d) sea ice concentrations (Comiso, 2017). Melt onset dates south of the mean ice edge are less reliable than those north of the edge. 

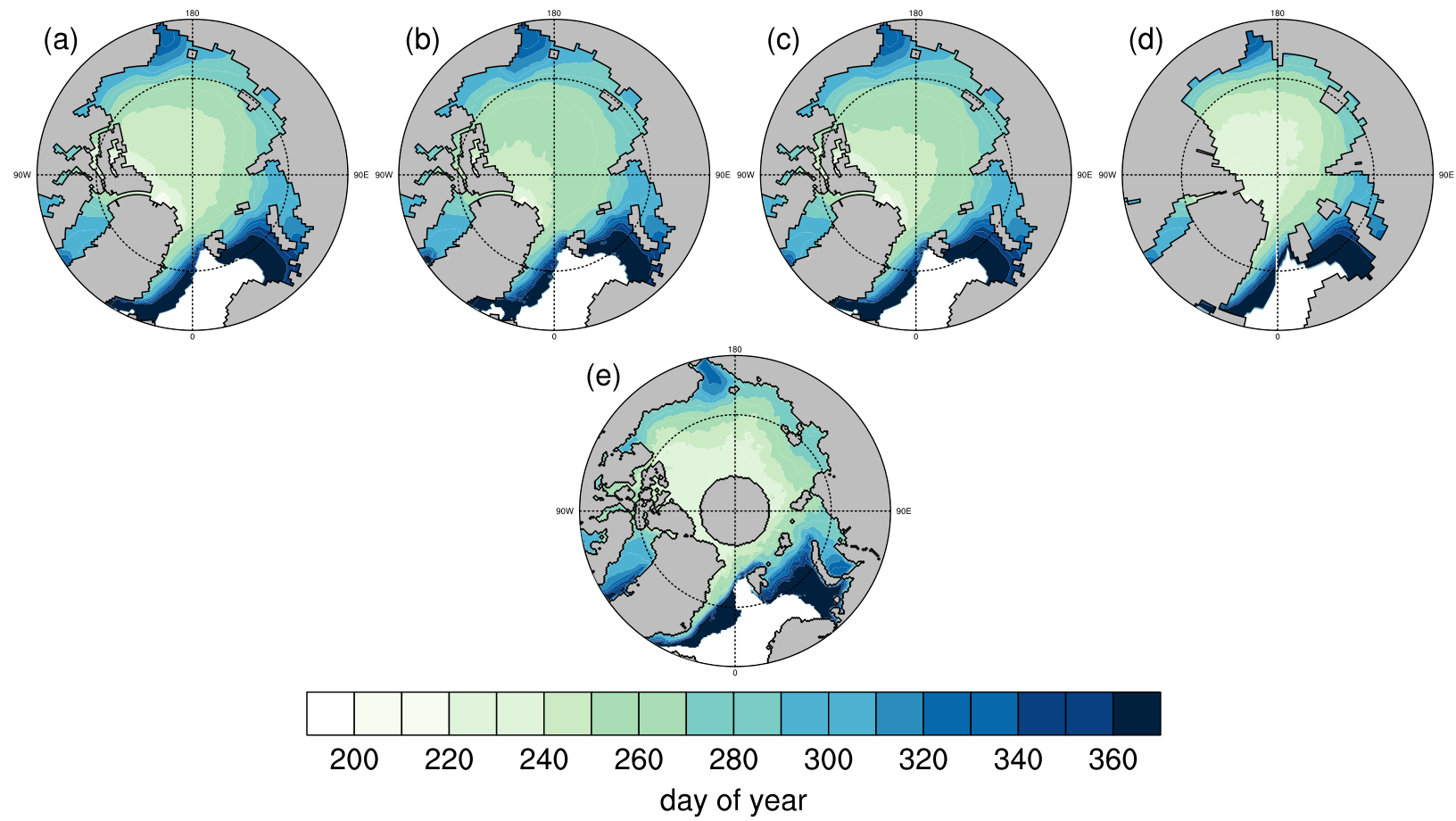

Figure S.4. Same as Fig. 4, but for ensemble member 34. Average freeze onset dates for 1979-2014 for each CESM LE definition using ensemble member 34: (a) congelation ice growth definition (b) frazil ice growth definition (c) thermodynamic ice volume tendency definition (d) surface temperature definition and (e) PMW satellite observations. 

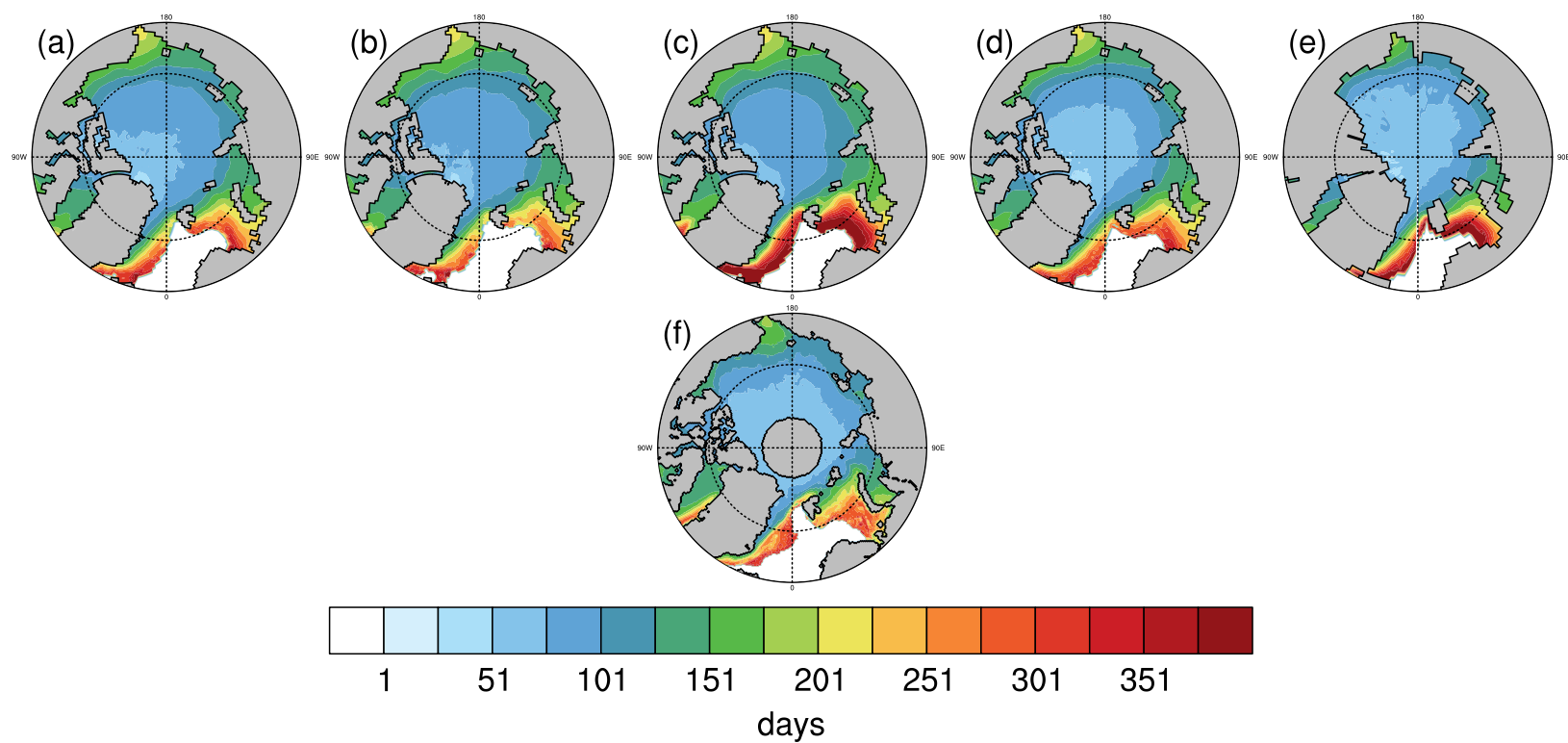

Figure S.5. Same as Fig. 5, but for ensemble member 34. Average melt season lengths over 1979-2014, for each CESM LE definition using ensemble member 34: (a) congelation-snowmelt (b) frazil-snowmelt (c) volume-volume (d) temperature-snowmelt (e) temperaturetemperature and (f) PMW satellite observations.
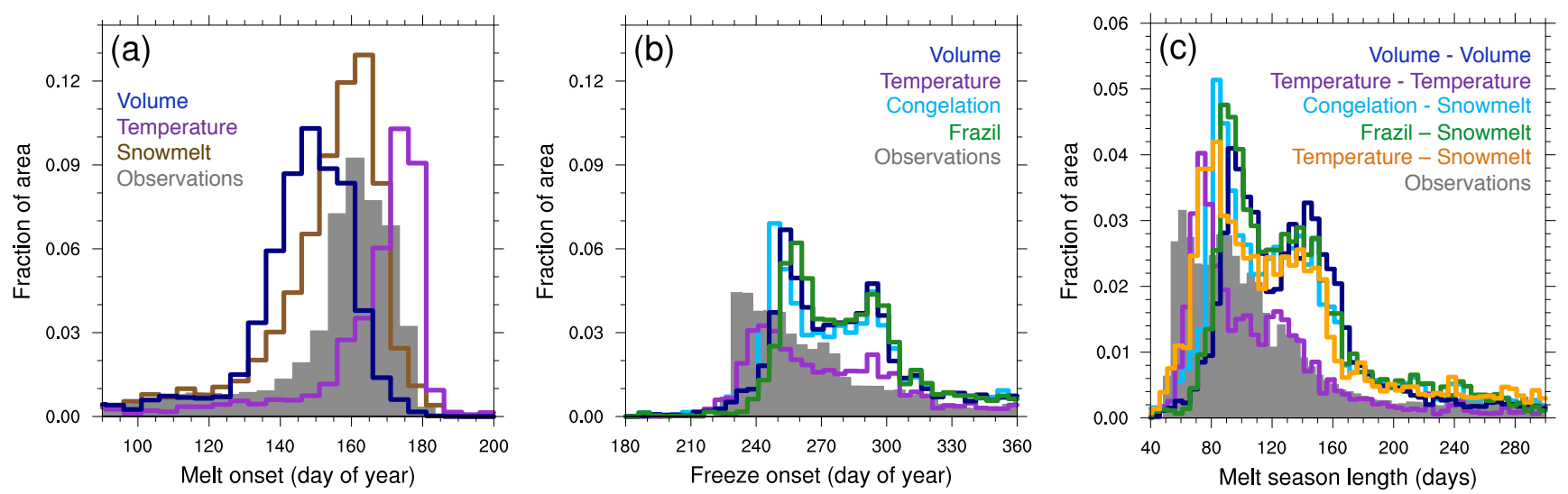

Figure S.6. Same as Fig. 10, but for ensemble member 34. Average melt season characteristics from $66^{\circ} \mathrm{N}$ to $84.5^{\circ} \mathrm{N}$ for $1979-2014$ for PMW satellite observations and each CESM LE definition (in ensemble member 34). (a) Melt onset using the surface temperature, thermodynamic ice volume tendency and snowmelt definitions (b) Freeze onset using the surface temperature, thermodynamic ice volume tendency, frazil ice growth and congelation ice growth definitions (c) Melt season length using the temperature-temperature, temperature-snowmelt, volumevolume, frazil-snowmelt and congelation-snowmelt definitions. 

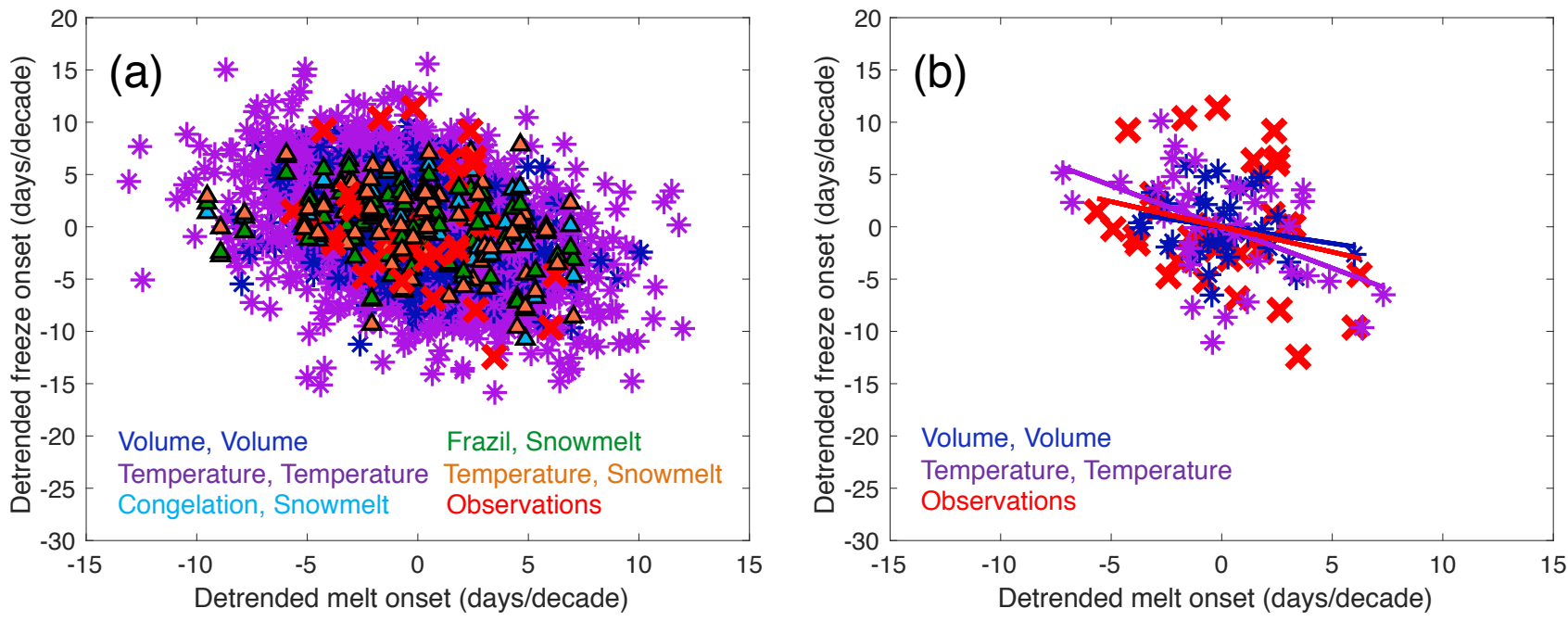

Figure S.7. Detrended pan-Arctic melt onset versus detrended pan-Arctic freeze onset for model definitions (different colors, see legend in figure) and PMW observations (X). Each marker represents one year in the period 1979-2014. (a) All ensemble members for all CESM LE definitions using temperature melt onset and temperature freeze onset, snowmelt melt onset and temperature freeze onset, volume melt onset and volume freeze onset, snowmelt melt onset and frazil freeze onset and snowmelt melt onset and congelation freeze onset (b) Ensemble means using temperature melt onset and temperature freeze onset and volume melt onset and volume freeze onset. Lines in (b) represent the least-squares linear fits. 

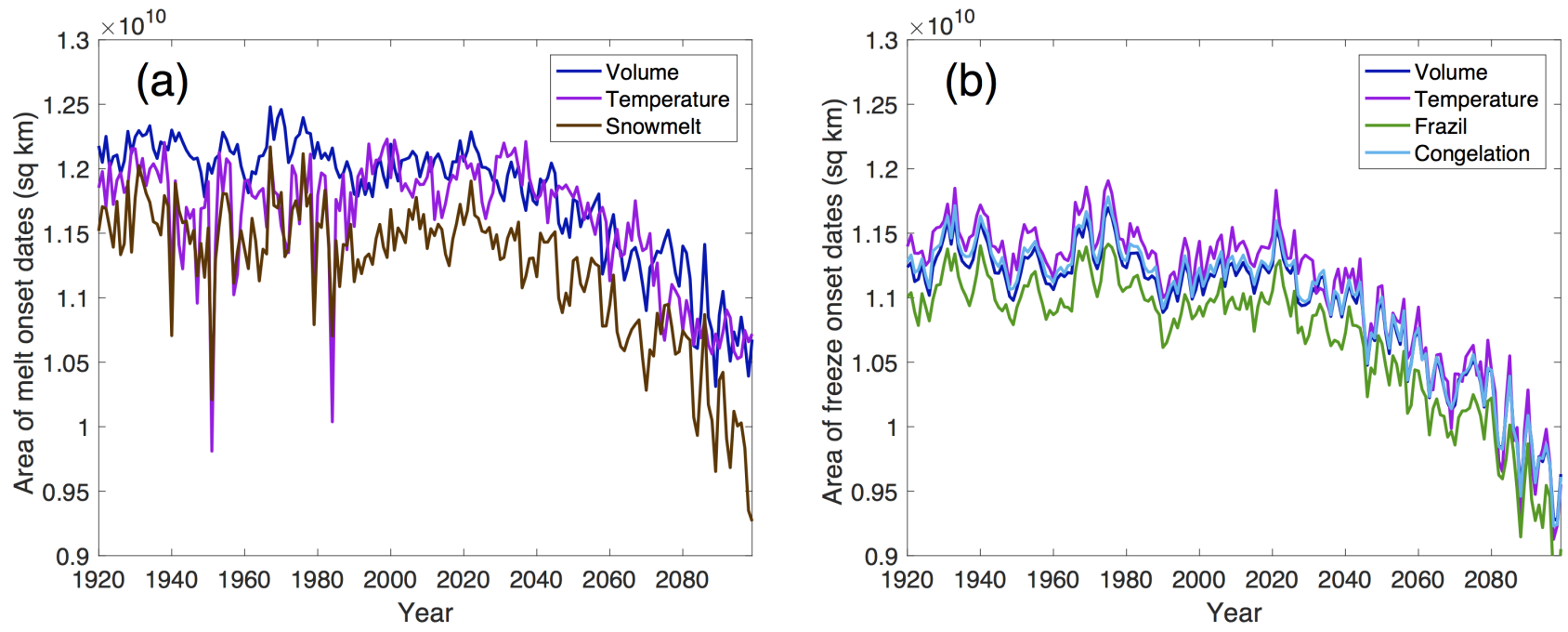

Figure S.8. Timeseries of the area covered by CESM LE melt (a) and freeze (b) onset dates [in $\mathrm{km}^{2}$ ] using ensemble member 35 , for the different definitions (different colors, see legend in figure). Due to different thresholds used for each definition, the areal coverage of melt and freeze onset dates are not necessarily the same for each definition in a given year. This is particularly apparent early on, with the areas becoming more similar at the end of the 21 st century. 